\title{
Generalized perpendicular bisector and circumcenter
}

\author{
Marc Rodríguez $^{1}$, Sere Abdoulaye ${ }^{2}$, Gaëlle Largeteau-Skapin ${ }^{1}$, Eric Andres ${ }^{1}$ \\ ${ }^{1}$ Laboratory XLIM-SIC, \\ University of Poitiers BP 30179, UMR CNRS 6712 \\ 86962 Futuroscope Chasseneuil Cedex, France \\ ${ }^{2}$ University of Ouagadoudou 03 BP 7021, Burkina Faso \\ \{rodriguez, glargeteau, andres\}@sic.univ-poitiers.fr
}

\begin{abstract}
This paper presents a theoretical generalization of the circumcenter as the intersection of generalized perpendicular bisectors. We define generalized bisectors between two regions as an area where each point is the center of at least one circle crossing each of the two regions. These new notions should allow the design of new circle recognition algorithms.
\end{abstract}

\section{Introduction}

Euclidean and discrete space have different behaviors. One of the principal issues in discrete geometry is to define discrete equivalents to Euclidean objects or transforms with as many useful (for a given application) properties as possible. In this paper, we are interested in the definition of a perpendicular bisector for discrete space. In Euclidean geometry, the intersection of the perpendicular bisectors of three points defines the circumcenter of the circumcircle. In this paper we are considering the problem of defining a perpendicular bisector adapted to discrete space for discrete circle recognition purposes. Classically, parameter space approaches are used for circle recognition [8]. The problem with these Hough transform type approaches is that there are three parameters (abscissa, ordinate of the center and radius) which makes it a three dimensional accumulator matrix. Various methods have been proposed to circumvent this problem [6]. These methods however are adapted for circle recognition in image analysis but not so much for problems such as invertible reconstruction. The discrete geometry community works for many years now on the problem of $2 \mathrm{D}$ and $3 \mathrm{D}$ invertible reconstruction of discrete objects. Discretization is the transform that associates a discrete object to a continuous one. Reconstruction is the transform that associates a continuous object to a discrete one. A reconstruction method is invertible if the discretization of the reconstructed object is equal to the original discrete object. Straight line segment recognition have been the main focus of research when it comes to invertible reconstruction $[13,14,1,4]$. There have been relatively few papers so far that could be used for invertible circle reconstruction [10]. 
Bisectors appear several times in the literature with usually a definition based on notion of equidistance. From the classical definition to discrete bisector functions which are used to analyze and filter medial axis $[3,12]$ where the medial axis of a Jordan curve is in any point equidistant to its borders. Bisectors between points and curves or between two curves have also been discussed in detail in the literature $[5,9]$ but to our knowledge, no definition for the bisector between two coplanar surfaces such as pixels has been proposed so far.

There are various ways of considering points in discrete geometry. In this paper, where we focus on dimension two, we consider that a discrete point is represented by a continuous surface element (pixel). We are therefore extending the notion of perpendicular bisector as the bisector between two regions. The generalized bisector of two $2 \mathrm{D}$ regions $A$ and $B$ is defined as the union of the perpendicular bisectors of all the couple of points $(p, q)$ where $p$ and $q$ are respectively points of the regions $A$ and $B$. This new definition extends the main property of the Euclidean perpendicular bisector of two points that is to be equidistant to both points. However, contrary to a Voronoi diagram, the generalized bisector is a surface and not simply a line as we will see. The new definition also extends the circumcircle property. For $n$ regions, the intersection of all the generalized bisectors corresponds to the center of all the circles that cross all $n$ regions. The definition is general and fits all type of regions and can be easily extended to higher dimensions. It gets however complicated very fast as illustrated in the paper with the generalized bisector of two disks which is a hyperbola. Even for pixels, as we will see, the generalized bisector is a complicated region bordered by straight line segments and pieces of parabolas. We propose a simplification that can be used for circle recognition.

The starting point of this paper is the definition of the perpendicular bisector between two regions. We examine the properties that are extended from usual perpendicular bisectors. In section three, we extend the circumcenter notion to surfaces, at first between three and after between an arbitrary number of surfaces. At the end of this section, we discuss what still needs to be done in order to obtain an efficient discrete circle arc recognition and reconstruction algorithm.

\section{The Perpendicular Bisector of two regions}

In this section, we focus on the perpendicular bisectors of two regions. We will present two approaches to define the generalized perpendicular bisector, the first is based on the notion of perpendicular bisector.

\subsection{Definition and Properties}

The idea is to extend the perpendicular bisector definition to regions. The perpendicular bisector of two points $p$ and $q$ in $\mathbb{R}^{n}$ corresponds to all the points that are equidistant from the two points. In $2 \mathrm{D}$, the perpendicular bisector is a 
straight line that is perpendicular to the straight line $p q$ and that passes through its midpoint. All the points of this perpendicular bisector are equidistant to both points $p$ and $q$ and each of them is therefore the center of a hypersphere that passes through both points. The idea here is to replace $p$ and $q$ by regions. This defines the generalized bisector (see Fig.1):

Definition 1 (Generalized perpendicular bisector). Let $\mathcal{S}_{1}$ and $\mathcal{S}_{2}$ be two regions. The generalized perpendicular bisector of $\mathcal{S}_{1}$ and $\mathcal{S}_{2}$ is the union of all the perpendicular bisectors of each couple of point $(X, Y)$ where $X \in \mathcal{S}_{1}$ and $Y \in \mathcal{S}_{2}$.

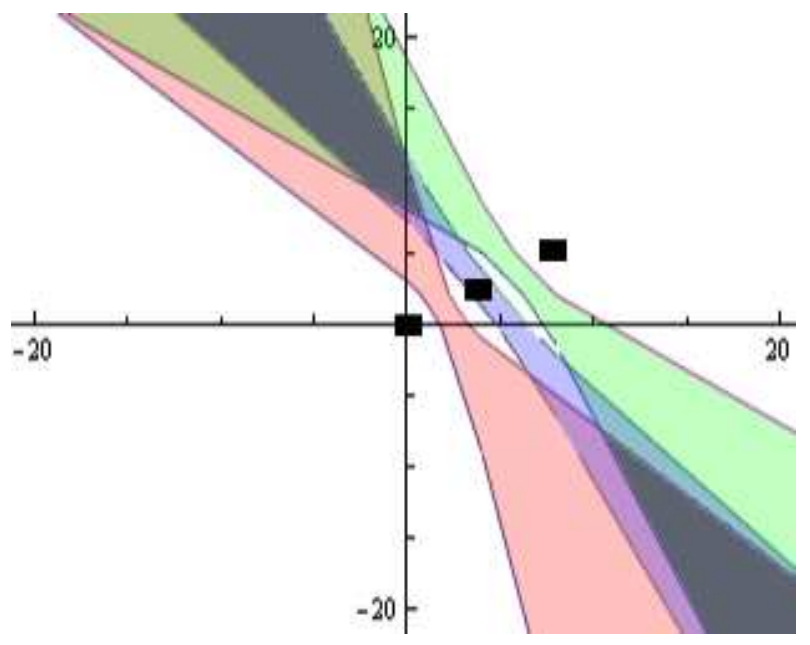

Fig. 1. A Euclidean couple of point has only one perpendiculat bisector but a couple of regions has an infinity of bisectors.

From this definition we can deduce two immediate properties:

Property 1: The generalized perpendicular bisector of two overlapping regions is the whole space even if they only share one point.

Proof. The bisector of two identical points is not defined if we consider the perpendicular line passing through the midpoint of the two points. If we consider the equidistance definition however it is easy to see that every point of the space is equidistant to both points.

Property 2: Each point of the generalized perpendicular bisector of two regions is the center of a circle crossing them.

This property is an immediate and direct consequence of the generalized bisector definition. 
The previous property leads to an alternative definition of the generalized bisector of two regions.

Definition 2 (Alternative definition of the generalized bisector). Let $\mathcal{S}_{1}$ and $\mathcal{S}_{2}$ be two regions. Let $d_{i}(X)=\min \left(d\left(X, \mathcal{S}_{i}\right)\right), D_{i}(X)=\max \left(d\left(X, \mathcal{S}_{i}\right)\right)$ where $d$ is the classical Euclidean distance.

Every Euclidean point $X \in \mathbb{R}^{n}$ such that the intervals $\left[d_{1}(X), D_{1}(X)\right] \cap\left[d_{2}(X), D_{2}(X)\right] \neq \emptyset$ belong to a region called the generalized perpendicular bisector of $\mathcal{S}_{1}$ and $\mathcal{S}_{2}$.

These two definitions are equivalent and yield to the same area. This second definition shows that, for each point of the generalized bisector, there is, in general, an interval of radii for which hyperspheres centered on the point will cut both regions.

\subsection{Generalized perpendicular bisector between two discs}

The generalized perpendicular bisector $(G P B)$ of two regions is not a straight line but an infinite set of straight lines that form a region. The shape of the generalized bisector depends on the shape of both regions.

For example, let $C_{1}=\left(O_{1}, r_{1}\right)$ and $C_{2}=\left(O_{2}, r_{2}\right)$ be two disks. The generalized bisector of both discs is a region bordered by a hyperbola (see Fig.2) defined by the asymptotes $d_{1}$ and $d_{2}$ and passing through $I$ and $J$. The straight lines $d_{1}$ and $d_{2}$ are the perpendicular bisectors of $t_{2}$ and $t_{1}$ which are the common tangents to both circles. $\overrightarrow{O_{1} I}=\frac{\left(1-r_{1}-r_{2}\right)}{2} \overrightarrow{O_{1} O_{2}}=-\overrightarrow{O_{2} J}$. As we can see, the $G P B$ is, in general, a complicated region.
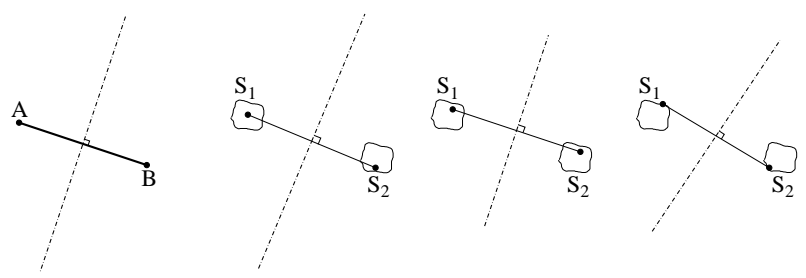

Fig. 2. Example of a generalized perpendicular bisector of two discs.

\subsection{Generalized perpendicular bisector between two pixels}

We are now interested in discrete spaces where each discrete point is represented a region classically called a pixel (a square region). While the generalized bisector is defined in arbitrary dimensions, we are focusing here on the dimension two. In the following, we explain how the generalized perpendicular bisector of two pixels can be piecewise computed. 
Let $P_{1}\left(x_{1}, y_{1}\right)$ and $P_{2}\left(x_{2}, y_{2}\right)$ be two pixels of which we want to compute the generalized bisector area. The pixel is the unit square region centered on a $\mathbb{Z}^{2}$ point $:\left\{(x, y), x \in\left[x_{i}-0,5, x_{i}+0,5\right]\right.$ and $\left.y \in\left[y_{i}-0,5, y_{i}+0,5\right]\right\}$. Let $X(x, y)$ be another point in $\mathbb{R}^{2}$. To know if the point $X$ is a good candidate to be in the generalized bisector area, we have to compute $d_{1}(X)=\min \left(d\left(X, P_{1}\right)\right)$, $D_{1}(X)=\max \left(d\left(X, P_{1}\right), d_{2}(X)=\min \left(d\left(X, P_{2}\right)\right)\right.$ and $D_{2}(X)=\max \left(d\left(X, P_{2}\right)\right)$.

We can see on figure 3 that the point of the pixel $P$ that is closest to $X$ can be either: $X$ itself if $X$ belongs to the pixel $P$, a point on an edge of $P$ if $X$ can be orthogonally projected on an edge of $P$ or a vertex of $P$ in all the other cases. The point $F$ of the pixel $P$ that is furthest from $X$ is always a vertex of $P$. Each pixel splits the space into 16 areas and so two non aligned pixels divide space in 49 areas where each area has a different formula for $d_{1}(X), d_{2}(X), D_{1}(X)$, and $D_{2}(X)$ (with of course symmetries).

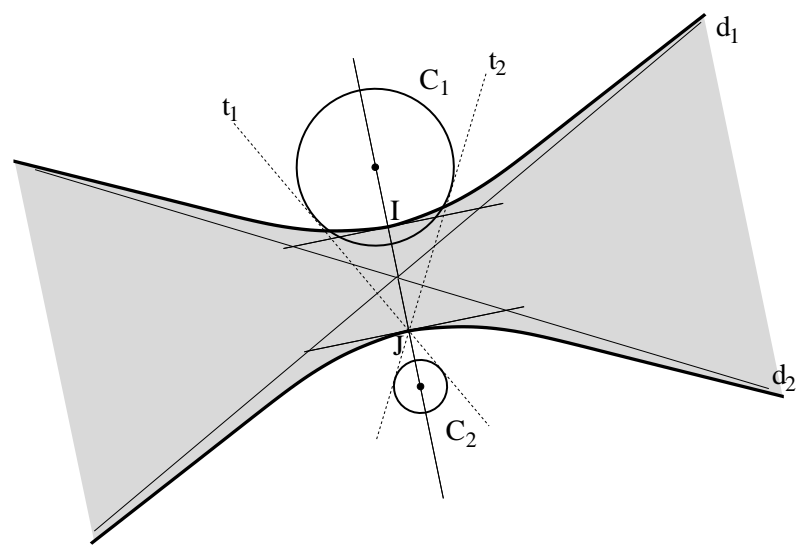

Fig. 3. $\mathrm{X}$ is a possible circle center if $\left[d_{1}(X), D_{1}(X)\right] \cap\left[d_{2}(X), D_{2}(X)\right] \neq \emptyset$.

Let us now examine what the intersection of intervals means in terms of equations. $\left[d_{1}(X), D_{1}(X)\right] \cap\left[d_{2}(X), D_{2}(X)\right] \neq \emptyset$ is equivalent to $\neg\left(\left[d_{1}(X), D_{1}(X)\right] \cap\left[d_{2}(X), D_{2}(X)\right]=\emptyset\right)$ which occurs only if $D_{1}(X)<d_{2}(X)$ or $D_{2}(X)<d_{1}(X)$.

Let $C_{i}\left(C_{i x}, C_{i y}\right)$ be the closest point of $X$ in the pixel $P_{i}$ and let $F_{i}\left(F_{i x}, F_{i y}\right)$ be the furthest point of $X$ in $P_{i}$. In each area, a constraint $d_{i} \leq D_{j}$ can be writen:

$$
\sqrt{\left(x-C_{i_{x}}\right)^{2}+\left(y-C_{i_{y}}\right)^{2}} \leq \sqrt{\left(x-F_{j_{x}}\right)^{2}+\left(y-F_{j_{y}}\right)^{2}}
$$

where $C_{i_{x}} \in\left\{x, x_{i}+0.5, x_{i}-0.5\right\}, C_{i_{y}} \in\left\{y, y_{i}+0.5, y_{i}-0.5\right\}, F_{j_{x}} \in\left\{x, x_{j}+\right.$ $\left.0.5, x_{j}-0.5\right\}$ and $F_{j_{y}} \in\left\{x, x_{j}+0.5, x_{j}-0.5\right\}$.

For the areas where $\mathrm{X}$ is not orthogonally projected on an edge of a pixel, we know that $x \neq C_{i_{x}}, x \neq F_{j_{x}}, y \neq C_{i_{y}}, y \neq F_{j_{y}}$. The two constraints can then 
be reduced to half planes inequations of type $\alpha x+\beta y+\gamma \leq 0$ where $\alpha, \beta, \gamma \in \mathbb{R}^{*}$.

For example, for the first case of Fig.3, we have $C_{1}=\left(x_{1}+0,5, y_{1}-05\right)$, $F_{1}=\left(x_{1}-0,5, y_{1}+0,5\right)$ and $C_{2}=\left(x_{2}-0,5, y_{2}+0,5\right), F_{2}=\left(x_{2}+0,5, y_{2}-0,5\right)$. The distance constraint $d_{1} \leq D_{2}$ is therefore :

$$
\begin{gathered}
\sqrt{\left(x-\left(x_{1}+0,5\right)\right)^{2}+\left(y-\left(y_{1}-05\right)\right)^{2}} \leq \sqrt{\left(x-\left(x_{2}+0,5\right)\right)^{2}+\left(y-\left(y_{2}-0,5\right)\right)^{2}} \\
=2\left(x_{2}-x_{1}\right) x+2\left(y_{2}-y_{1}\right) y+\left(x_{1}^{2}-x_{2}^{2}+x_{1}-x_{2}+y_{1}^{2}-y_{2}^{2}-y_{1}+y_{2}\right) .
\end{gathered}
$$

In the other areas, we are at least in one of this cases $C_{i_{x}}=x, F_{j_{x}}=x, C_{i_{y}}=$ $y$ or $F_{j_{y}}=y$. There are no simplifications during the calculation, the constraints correspond to pieces of parabolas of the form: $(x-\alpha)^{2}+(y-\beta)^{2}-\gamma \leq 0$.

\subsection{Approximation of the generalized bisector for two pixels}

To simplify the description of the generalized perpendicular bisector of two pixels, we are going simply to ignore the pieces of parabolas in the piecewise description of the generalized bisector. The pieces of parabolas are actually very small (not larger than a pixel). We consider here that each pixel divides space into only four areas by doing as if the closest point of a pixel is always one of the vertices. The approximated generalized perpendicular bisector border is therefore only composed of straight line pieces (see fig.4).

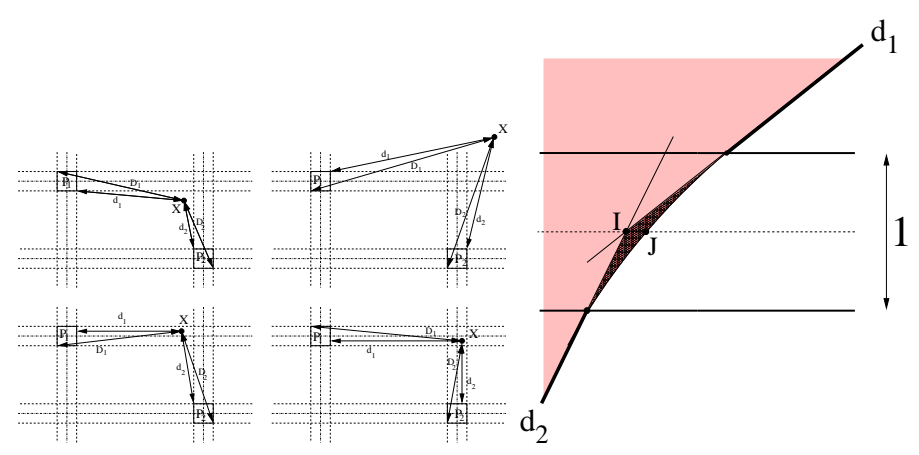

Fig. 4. On the left, the exact perpendicular bisector and the approximation where the parabolic pieces have been dropped by extending the straight lines. The generalized bisector is slightly reduced.

With this approximation, we reduce (very slightly) the solution space (the possible circle centers). If a circle is recognized with help of the simplified generalized bisectors it means that it is also a solution to the generalized bisector. We might reject some valid solution and not recognize a valid circle but since the 
approximation is so minimal, this would probably fall into the margin of error of the computations anyway.

\section{The generalized circumcenter}

The Euclidean circumcenter of a set of Euclidean points is defined by the intersection of all the bisectors from pairing the points two by two. Its main property is to be the center of the circle passing through all the points of the set. In this section, we naturally extend this notion to $2 \mathrm{D}$ regions.

Definition 3 (Generalized Circumcenter $(G C)$ of a set of regions). The generalized circumcenter $(G C)$ of set of $n$ regions $\mathcal{S}=\left(S_{i}\right)_{i \in[1, n]}$ is the intersection of the generalized perpendicular bisectors $(G P B)$ of every two regions of the set:

$$
G C(\mathcal{S})=\bigcap_{i, j \in[1, n], i<j}\left(G P B\left(S_{i}, S_{j}\right)\right) .
$$

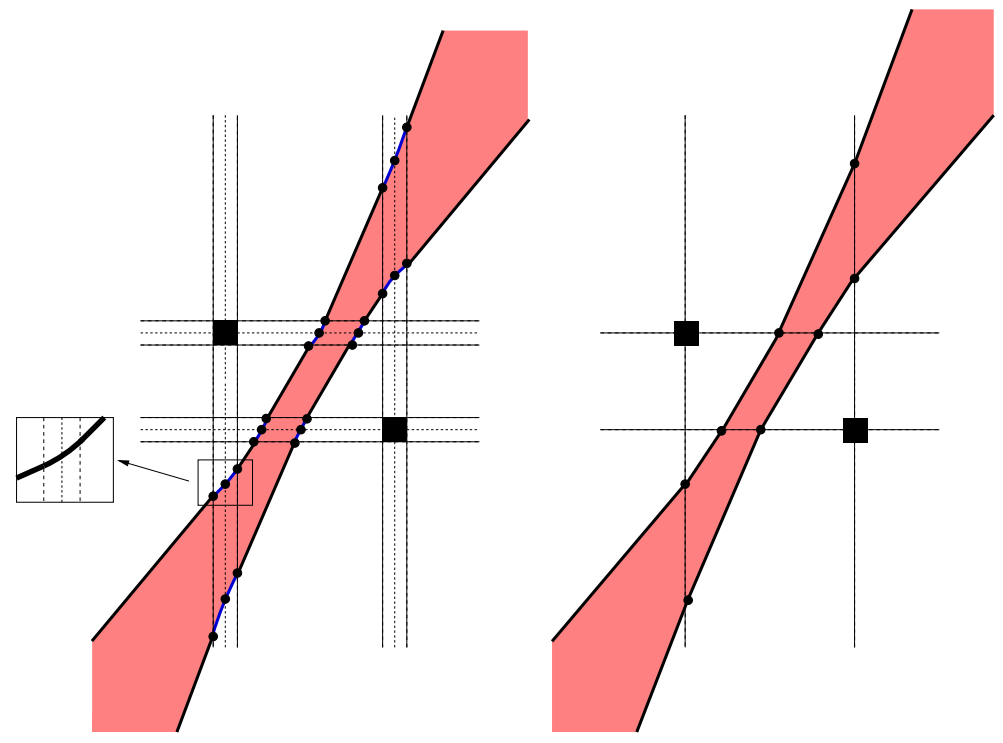

Fig. 5. Generalized circumcenter of $P_{1}=(0,0), P_{2}=(2,6)$ and $P_{3}=(6,3)$.

We can see on figure 5 the generalized circumcenter of three pixels and on figure 6 the generalized circumcenter of four pixels.

For the Euclidean circumcenter, if the points are aligned they belong to a Euclidean straight line which forms a circular arc of an infinite radius. In discrete space things are a little bit different as we can see on figure 7: discrete straight 


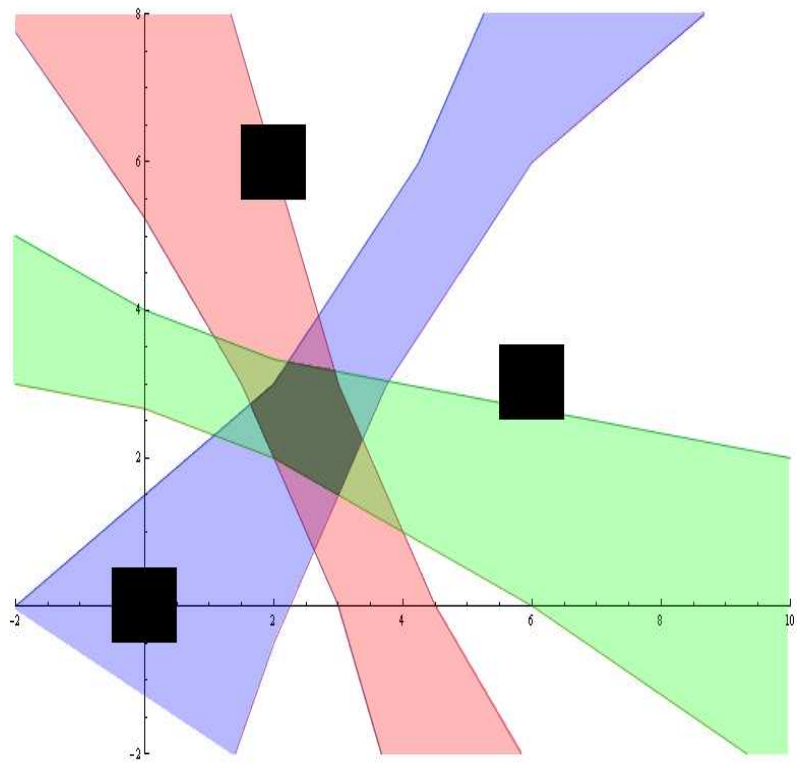

Fig. 6. The generalized circumcenter of $\{(0,0),(2,11),(6,2),(8,6)\}$.

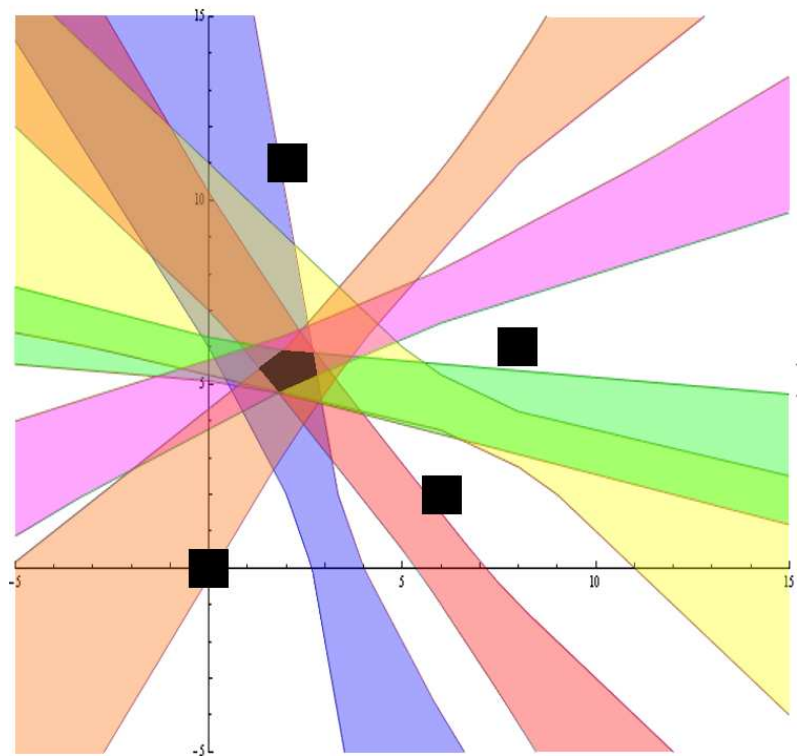

Fig. 7. when the pixels are aligned, the generalized circumcenter (dark region) is not connected. The pixels are marked in black. 
line segments belongs to discrete circles. The generalized circumcenter is, in this case, a disconnected infinite region.

One of the last remaining question is the existence of a general solution. All the points in the generalized circumcenter of a pixel set are the center of at least one circle crossing every two pixel of the set. That does not mean that there exists a circle that crosses every pixels of the set. We need to ensure that a common radius exists. Actually, there always exists a solution:

Theorem 1 (Circle existence). If the generalized circumcenter $G C(\mathcal{S})$ of a set of regions $\mathcal{S}$ is not empty, then for all $X \in G C(\mathcal{S})$ there exists at least one circle centered in $X$ that crosses all the regions of the set $\mathcal{S}$.

Proof. This result is the direct application of Helly's theorem [7]. The radius dimension is 1 . The generalized circumcenter of a set of regions is the intersection of the generalized circumcenters for every two regions of the set. This defined each time a non empty interval of radii for every two regions of the set.

Note that if the circumcenter area is empty then there is no circle that intersects all the regions. This is not completely true if we use the simplified generalized perpendicular bisector. The area of solutions that are neglected are however, as we have already stated, very small.

\section{Conclusion and Perspectives}

In this paper, we have proposed a generalization of perpendicular bisectors and circumcenters to regions in an arbitrary dimensional space. This extension is very interesting because many properties from classical segment bisectors are preserved. We have shown that, in $2 \mathrm{D}$, all the points of the generalized circumcenter of a set of regions are the center of at least one circle that crosses all the regions of the set. We have described the generalized bisector of two pixels. We have also proposed a slight simplification of the generalized bisector of two pixels in order to avoid the pieces of parabola on its border.

The generalized circumcenter is a new interesting approach to digital circles recognition and reconstruction because it contains all the centers of all the Euclidean circle that pass through all the pixels. It can be used for full discrete circle recognition or only for discrete circular arc recognition $[2,11]$. The generalized perpendicular bisector is easily defined in upper dimensions. The $n$ dimensionnal circumcenter of a voxel set will be a volume containing the centers of all the Euclidean hypersphere crossing all the voxels of the set. One of the last advantages of this approach is that the pixels/voxels in the recognition process do not need to all be of the same size. This could allow multiscale or noisy circle/hypersphere recognition.

Much work remains to be done however. There are some complicated algorithmic problems that need to be solved before an efficient circle recognition algorithm can be proposed. The intersection of the simplified generalized bisectors is algorithmically costly because (despite the fact that they are defined by 
straight lines) they are not convex. Secondly, for $n$ regions we have $O\left(n^{2}\right)$ generalized bisectors to intersect in order to compute the generalized circumcenter. We are looking right now into some simplification in the recognition process that should allow us, at least, to be able to fast reject cases where there are no solutions. The long term goal of all this is to be able to have an invertible circle recognition algorithm that can be paired with straight line recognition algorithms.

\section{References}

1. R. Breton, I. Sivignon, M. Dexet, and E. Andres. Towards an invertible euclidean reconstruction of a discrete object. In Discrete Geometry and Computer Imagery, volume LNCS vol. 2886, pages 246-256, 2003.

2. David Coeurjolly, Gerard Yan, Jean-Pierre Reveills, and Laure Tougne. An elementary algorithm for digital arc segmentation. Discrete Applied Mathematics, 139(1-3):31-50, 2004.

3. M. Couprie, D. Coeurjolly, and R. Zrour. Discrete bisector function and euclidean skeleton in 2d and 3d. 25(10):1543-1556, October 2007.

4. M. Dexet and E. Andres. A generalized preimage for the digital analytical hyperplane recognition. Discrete Applied Mathematics, 157(3):476-489, 2009.

5. R. T. Farouki and J. K. Johnstone. Computing point/curve and curve/curve bisectors. In R. B. Fisher, editor, The Mathematics of Surfaces V, pages 327354. Oxford University, Oxford, 1994.

6. R.C. Gonzalez, R.E. Woods, and S.L. Eddins. Digital Image Processing Using $\operatorname{MATLAB}(R)$. Prentice Hall, 2004.

7. E. Helly. Uber mengen konvexer korper mit gemeinschaftlichen punkten. In Jahresbericht der Deutschen MathematikerVereiningung, volume 32, pages 175-176, 1923.

8. D. Ioannou, W. Huda, and A.F. Laine. Circle recognition through a $2 \mathrm{~d}$ hough transform and radius histogramming. 17(1):15-26, January 1999.

9. M. Peternell. Geometric properties of bisector surfaces. 62(3):202-236, 2000.

10. T. Roussillon, L. Tougne, and I. Sivignon. On three constrained versions of the digital circular arc recognition problem. pages 34-45, 2009.

11. Tristan Roussillon, Isabelle Sivignon, and Laure Tougne. On-line Recognition of Digital Circular Arcs. In 15-th IAPR International Conference on Discrete Geometry for Computer Imagery (DGCI), LNCS. Springer, September 2009.

12. H. Talbot and L. Vincent. Euclidean skeletons and conditional bisectors. 1818:862876, 1992.

13. J. Vittone and J.-M. Chassery. (n,m)-cubes and farey nets for naive planes understanding. In Discrete Geometry for Computer Imagery, volume LNCS vol. 1568, pages 76-90, 1999.

14. J. Vittone and J.-M. Chassery. Recognition of digital naive planes and polyhedrization. In Discrete Geometry for Computer Imagery, volume LNCS vol. 1953, pages 296-307, 2000. 